\title{
Buckwheat may Help Control Satiety and Reduce Risk Factors for Type 2 Diabetes
}

\author{
Stephanie Caligiuri \\ (University of Manitoba) \\ News Reporter (HSI 2011-2012)
}

A balanced diet containing functional foods can play a crucial role in preventing one of the most prevalent diseases in Canada, type 2 diabetes. Research on functional foods has increased tremendously over the last decade; functional foods contain components that provide health benefits, such as reducing chronic disease risk, above what typical nutrients can offer. Researchers at the University of Manitoba and Canadian Centre for Agri-Food Research in Health and Medicine have investigated a potential role for functional foods, specifically buckwheat, in reducing the risk for type 2 diabetes.

Excess body fat is one of the strongest risk factors for type 2 diabetes, with the Canadian Diabetes Association reporting that $80-90 \%$ of patients with type 2 diabetes are overweight or obese. ${ }^{1}$ In an effort to prevent this susceptible population from developing type 2 diabetes, several researchers have focused on dietary interventions that may help control hunger, blood sugar levels, and body weight.

\section{In an attempt to prevent the development of type 2 diabetes, several researchers have focused on dietary approaches which may help control hunger, blood sugar levels, and body weight.}

Danielle Stringer, a PhD candidate at the University of Manitoba, is currently investigating the beneficial properties of buckwheat. Buckwheat is similar to a cereal grain and is quite versatile as it can be incorporated into soups, porridge, salad, or flour. Buckwheat is low in fat and high in fibre and protein.

Buckwheat seeds are a very affordable functional food $(\$ 0.70 / 100 \mathrm{~g})$, making it a cost-effective dietary intervention. Given that individuals of lower socioeconomic statuses have a higher risk of developing type 2 diabetes, ${ }^{4}$ buckwheat may be a viable food choice to enhance health

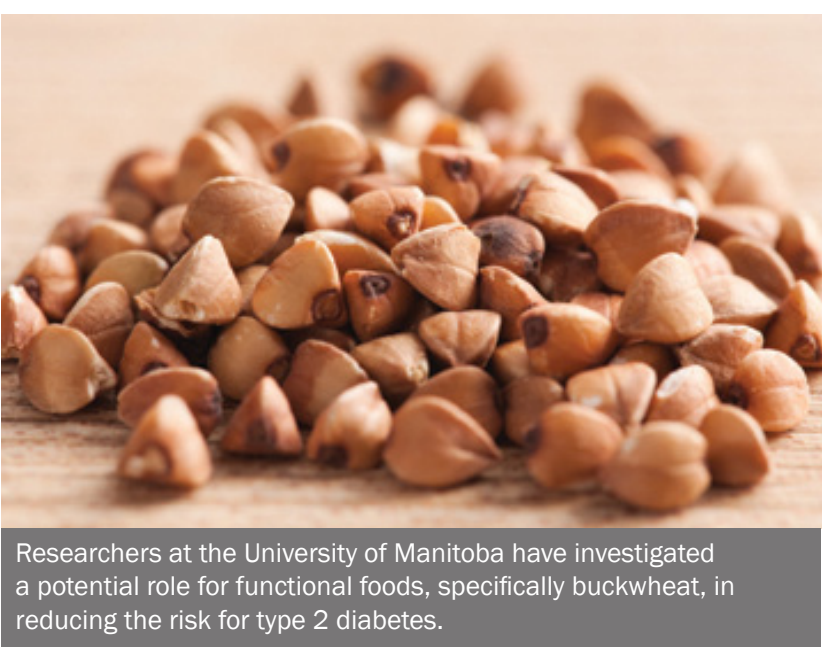

in lower socioeconomic groups.

Stringer stated, "I wanted to research the effect of functional foods on diabetes because there is great research potential for nutrition interventions and improving the health and quality of life for patients with diabetes. I performed a study on buckwheat in particular because my lab has produced results both in vitro and in animal studies that showed the potential of buckwheat in lowering blood glucose levels and potentially altering satiety. Buckwheat is thought to contain insulin-like compounds that has the potential to maintain blood sugar levels and reduce the incidence of type 2 diabetes."

Other researchers also interested in buckwheat have reported beneficial effects. Zhang et al. (2007) found that the rate of abnormal blood glucose levels was $1.9 \%$ in Mongolian-Chinese participants who consumed buckwheat seeds compared to $7.3 \%$ in those who did not. ${ }^{2}$ In addition, the researchers found that those who consumed buckwheat had significantly lower levels of triglycerides, cholesterol, and low density lipoprotein cholesterol (bad cholesterol) than the non-buckwheat-consuming population. ${ }^{2}$ Therefore, buckwheat may provide additional benefits for the promotion of health.

Animal studies provide additional evidence for the favourable effects of buckwheat. A study performed by 
Kawa et al. (2003) found that buckwheat concentrate (10 and $20 \mathrm{mg} / \mathrm{kg}$ body weight) effectively lowered blood glucose by $12-19 \%$ in diabetic rats. ${ }^{3}$

Stringer is currently performing research on buckwheat and its potential role in aiding type 2 diabetes patients with blood glucose control and satiety. Stringer wants to further investigate the correlations reported by Zhang and Kawa using in vitro studies and randomized controlled clinical trials.

Curran and Stringer (2010) sought to identify the mechanism that allows buckwheat to stabilize blood glucose levels using H411E rat hepatoma cells. Curran and Stringer hypothesized that the compounds D-chiro-inositol and myo-inositol in buckwheat would have insulin mimetic properties. They tested a buckwheat concentrate, D-chiro-inositol and myoinositol separately. The buckwheat concentrate had insulin mimetic effects on protein phosphorylation events during glucose uptake and metabolism; however the inositol compounds were not responsible for these effects. Curran and Stringer concluded that buckwheat has insulin-like effects in controlling blood glucose levels and that those effects must be attributable to compounds in buckwheat other than the inositols. ${ }^{5}$

Stringer has also investigated the effect of buckwheat in humans. Her study included 12 healthy participants and 12 patients with diabetes who controlled their blood glucose levels through diet alone. She produced crackers using buckwheat flour and asked participants to consume $75 \mathrm{~g}$ of either buckwheat crackers or rice crackers (control) in a fasted state. The $75 \mathrm{~g}$ dose of buckwheat crackers contained $46 \mathrm{~g}$ of whole grain buckwheat flour. Gastrointestinal hormones that can affect glucose metabolism such as glucagon like peptide-1 (GLP-1), gastric inhibitory polypeptide (GIP), as well as hormones responsible for controlling satiety such as ghrelin, peptide $Y Y$, and pancreatic polypeptide, were analyzed after consuming the crackers.
Stringer noted, "the buckwheat crackers increased the secretion of hormones associated with satiety in both the healthy control group and the patients with type 2 diabetes." The changes in hormone secretion can translate to reductions in appetite and improved eating patterns. With longer feelings of fullness, individuals will be less likely to eat more and will also be more likely to obtain normal blood glucose levels. This will also aid individuals in achieving and maintaining a healthy body weight, which can help to reduce their risk of developing type 2 diabetes.

Stringer concluded, "There lies great potential with buckwheat products for patients with diabetes to help maintain their body weight and glucose control. The implications of this research can translate into the incorporation of the active ingredients of buckwheat into a variety of food products, which can offer consumers a costefficient means to aid in the prevention or management of type 2 diabetes."

\section{Acknowledgements}

Special thanks to Danielle Stringer, PhD candidate, for her time and willingness during the interview.

\section{References}

1. Canadian Diabetes Association. Canadian Diabetes Association 2008 clinical practice guidelines for the prevention and management of diabetes in Canada. Canadian Journal of Diabetes. 2008: 32; suppl 1-201.

2. Zhang HW, Zhang YH, Lu MJ, Tong WJ, Cao GW. Comparison of hypertension, dyslipidaemia and hyperglycaemia between buckwheat seed-consuming and non-consuming Mongolian-Chinese populations in Inner Mongolia, China. Clin Exp Pharmacol Physiol. 2007 Sep;34(9):838-44.

3. Kawa JM, Taylor CG, Przybylski R. Buckwheat concentrate reduces serum glucose in streptozotocin-diabetic rats. J Agric Food Chem. 2003; 51:72877291.

4. Statistics Canada, Catalogue no. 82-003-XPE - Health Reports, Vol. 21, no. 3, September 2010, 14-year diabetes incidence: The role of socio-economic status. Research article.

5. Curran JM, Stringer DM, Wright B, Taylor CG, Przybylski R, Zahradka P. Biological response of hepatomas to an extract of Fagopyrum esculentum M. (Buckwheat) is not mediated by inositols or rutin. J Agric Food Chem. 2010; 58:3197-3204.

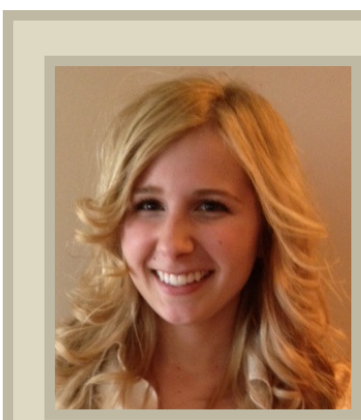

\section{Stephanie Caliguri}

Stephanie Caligiuri is completing her Master of Science degree in Human Nutritional Sciences at the University of Manitoba. Her research has focused on the pathophysiology of obesity related kidney disease and how interventions with altered dietary fatty acid compositions may ameliorate or accelerate the disease. Stephanie plans to start her PhD in the Faculty of Medicine this Fall in which she will be running a clinical trial investigating the influence of flaxseed and flax oil on vascular health and hypertension. Ms. Caligiuri aspires to be a researcher focusing on using functional foods and natural health products as treatment/preventive measures for chronic disease. 